\title{
The New Bridge Converter Control Method
}

\author{
R. M. Radetic ${ }^{1}$, D. R. Milivojevic ${ }^{2}$, M. Pavlov ${ }^{3}$ \\ ${ }^{1}$ Serbian Transmission System, Nade Dimic, 40, 19210, Bor, Serbia, rradetic@ptt.rs \\ ${ }^{2}$ Mining and Metallurgy Institute, Zeleni bulevar, 35, 19210, Bor, Serbia, dragan.milivojevic@irmbor.co.rs \\ ${ }^{3}$ Mining and Metallurgy Institute, Zeleni bulevar, 35, 19210, Bor, Serbia, marijana.pavlov@irmbor.co.rs
}

The article describes a new control method of transistor power bridge converter. It is a new one, because of some original solutions and its difference in comparison to existing methods. It's irrelevant of load, similar to the "phase shifted" control method. It is easy to realize using ordinary electronic elements. A significant advantage of method is the possibility of permanent output current monitoring by measuring the voltage sag on a pair of transistors. That allows a very efficient current protection.

Keywords: Control method, Bridge converter, PWM

\section{INTRODUCTION}

$\mathrm{T}$ HE TRANSISTOR bridge power converters are in use by DC/DC converters (chopper type E, [1], [2]) and by $\mathrm{DC} / \mathrm{AC}$ converters (invertors) as well. The bridge structure consists of four transistors, as it is shown in Fig.1. The direct current input supply voltage is $U_{\mathrm{B}}$.

The control, automated regulation, can be realized by use of voltage, or current.

In the case of voltage control, the output voltage is compared with the reference voltage, and the difference generates the control pulses for the transistors. The fast supply voltage variations, or the changes on the loading side, are detected just as an effect on output signal. This makes the regulation slow.

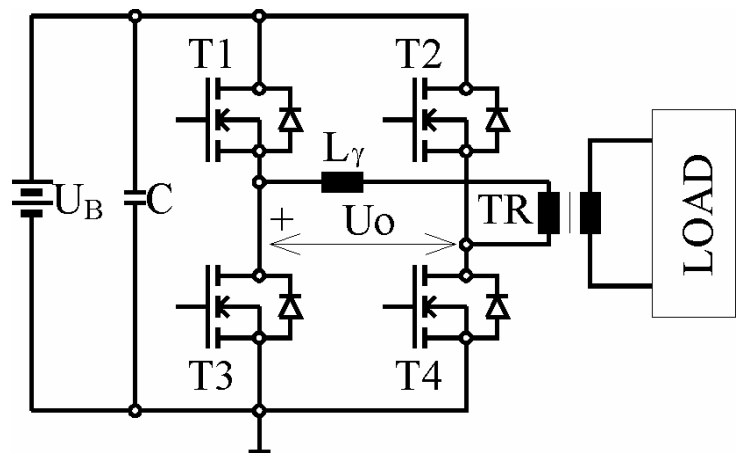

Fig.1 The simplified circuit diagram of a transistor bridge converter

The current feedback is faster. There are two ways of control: the mean current value and current spikes. The second one is very important. It saves the converter during sharp changes of voltage or in case of short circuit. The current sensing is possible to obtain by sensors (current transformer and diode bridge, Hall element, shunt etc.). The measuring of voltage variations on the transistors as the consequence of current variations can be used for current detection. In that case there is no need for any sensors, and the design and realization are simpler.

The output voltage regulation in $\mathrm{DC} / \mathrm{AC}$ conversion is done by pulse width modulation (PWM). Mainly two ways are used to generate PWM pulses: classical and "phase shifted" method.

\section{Some of The Usual Control Methods}

The classical method provides the simultaneous switching on and off of the diagonal transistors, Fig 2. The control PWM voltage produces the same form of output PWM when the pure resistance loading is connected only.

When inductive load is connected to the output and all transistors are switched off, the energy of the inductance is transferred back to the power source via freewheeling diodes.

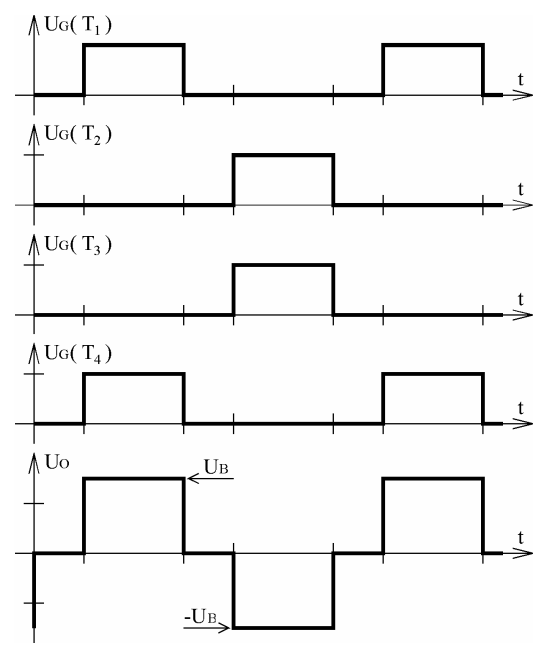

Fig.2 The classical method voltage diagram

After that, the primary transformer is not connected to the terminals of power source anymore, that means it is free. Parasitic output voltage oscillations are occurring because of parasitic capacitance. The oscillations are reduced by implementation of special snubber circuits that are increasing the converter power dissipation. Use of snubber circuit increases switching losses of the transistors, although there is a very simple way of regulation. If the control voltages for transistors are transferred via transformer, only one driver transformer is needed. This kind of control is useful when the pure resistance or a rectifier is connected to the bridge output or transformer secondary.

The "phase shifted" method is a better way of regulation. In that case, two bridge halves are separately controlled. The 
duty cycle (D) for both halves of the bridge is $D=0.5$, but for the second half it is phase shifted. When both bridge sides are in-phase (phase-locked) they produce equal outputs and the difference is zero. If the phase of the second side is moved, the voltage on the connected load grows. The maximal value of output voltage is by phase difference of $180^{\circ}$. This control method is more complex than the previous one. It is realized by special circuits (UC3875, UCC3895, for example).

If the main inductive load is connected to the output, the accumulated energy remains in the circuit, rather than to transfer back to the source. At that time, the current circuit is closed via one of the transistors in one bridge half and the appropriate diode in the other half. The transistor and diode of the positive group change those of the negative one and vice versa (Fig.3). It makes current monitoring by transistor voltage drop measuring more complicated. The most simple way is to do that on the transistors $\mathrm{T}_{3}$ and $\mathrm{T}_{4}$ (Fig.1) because their sources (S) are on the same, common potential.

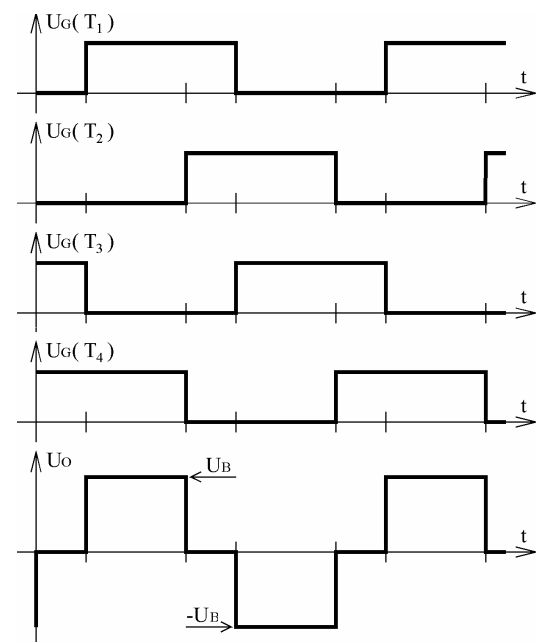

Fig.3 "Phase shifted" method control voltage diagram

Except for these typical methods, there are some alternative solutions. One of them is to use $\mathrm{D}=50 \%$ for noncontrolled transistors and to control the other two transistors by PWM. It could be the upper or the lower pair of transistors. Such method is used by Intersil [4] in their controllers ISL6551 or ISL6752. Concretely, the upper pair is running by $\mathrm{D}=50 \%$ and the lower one is PWM controlled.

The other way of control is described in [3]. One half of the bridge is driven by PWM and the other one uses $\mathrm{D}=50 \%$.

But the bridge transistor power converter control method, which will be explained below, is slightly different from any described above.

\section{The New - COMPLEMENTARY CONTROL Method}

This control method is based on driving both bridge halves separately by two complementary control voltages with phase difference of $180^{\circ}$, Fig. 4 a). The upper (positive) bridge half is controlled by PWM control voltage and the bottom one (negative half) by inverted control voltage. The control PWM voltage is generated by one of standard PWM controllers. Its complementary voltage is produced by inversion itself. The "dead time" (delay) must be introduced to prevent a situation where both transistors in the same half of the bridge are switched on and conduct simultaneously [5].
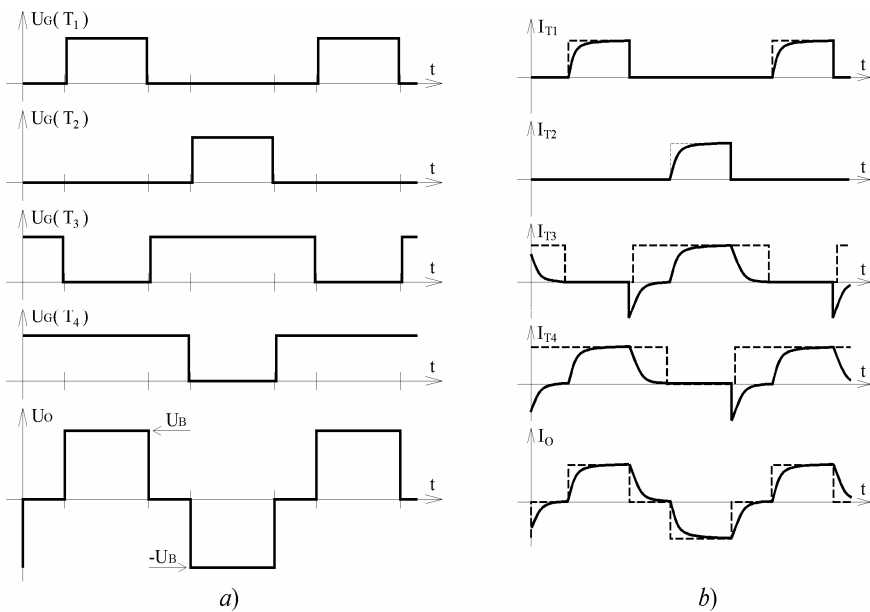

Fig.4 The control voltage and output current diagrams

It is totally irrelevant which part of bridge will be controlled by PWM signal, upper or lower one. But, related to output current monitoring, it is convenient that the control PWM signal leads to the upper pair of transistors, and complementary voltage to the lower pair. The example of that solution is shown in Fig.5. If the bridge converter is realized by MOSFET transistors, the solution may be more efficient, because in some time intervals the current can flow through the MOSFET channel (in opposite direction) instead of through the diode. In that case, the control pulses generation is quite simple. The PWM pulses are lead to the upper transistors (T1 and T2) via drivers. The control pulses for the lower bridge part are generated by inversion of the previous signal and introducing the "dead time". Most drivers have both of those functions. The typical example for PWM pulse generation is SG3525 circuit and IR2111 driver. Regarding Fig.1, both transistor connections (T3 and T4) are on the common minus (-) potential of power source. One of the transistors is switched on at all times. When the transformer primary voltage (the bridge output) is zero, both transformer connections are approximately the same on the potential as the power source minus pole.

Prevalent inductive bridge output load means that the current flows through one of the transistors and the other site diode.

\section{REALIZED COMPLEMENTARY CONTROL UNIT}

Fig. 5 shows the overal diagram of an example of a control circuit. The integrated module SG3525 generates PWM control pulses in accordance with regulation voltage produced by control logic. These two modules are galvanically isolated. The regulation voltage can contain a current regulation from current feedback. The driver circuits IR2111 are driven only by one pulse output of SG3525 that is lead to the upper transistors in the bridge. The control voltage for the lower transistors is produced by inversion and addition of "dead time". The dead time interval is $700 \mathrm{~ns}$ for that specific circuit. The fast and immediate overcurrent protection is achieved by monitoring voltage variations across the lower pair of 
transistors. In concrete case it is done via diodes D2, D3, D4 and D5 and resistances R3, R4 and R5, Fig.5. The current monitoring is provided by measuring of saturated voltage of IGBT, or voltage between source and gain $\left(\mathrm{U}_{\mathrm{DS}}\right)$ of MOSFET transistors. The very fast overload appearance (or shortcircuit) disables the control pulses and switches off the transistor momentarily via pin 10 of SG3525, Fig.5.

All these activities take not more than $1 \mu \mathrm{s}$. It should be considered as a momentary overcurrent protection. The quick overcurrent protection, which shortens the consecutive pulses - "soft start", could be achieved by discharging capacitor C10 via transistor $\mathrm{T} 1$ in control logic circuit. Those details are not shown in Fig.5.

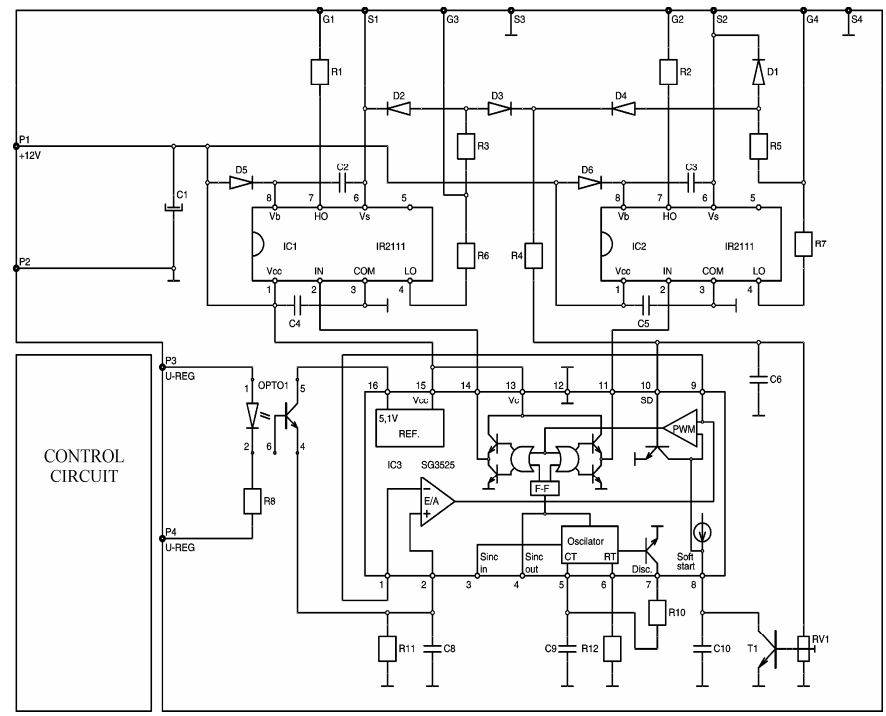

Fig.5 The circuit diagram of the new complementary control method

\section{CONCLUSION}

The described method of transistor power bridge converter control is interesting because of its specific way of operation. This method enables to obtain the same voltage shape as with "phase shifted" type of control.

The main advantage is the simple realization using available low cost components. The complete current supervision is improved and accomplished without additional current sensors. It is achieved by observation of voltage drop across the lower transistors only. There are no intervals when transistors $\mathrm{T} 1$ and $\mathrm{T} 2$ are on at the same time. It is also the method advantage.

The new control concept has been introduced and experimentally verified.

Using this method the $3 \mathrm{~kW}$ power converter has been created, Fig.6. The IGBT type transistors are in use on the bridge output. The signal power is slightly increased, and a circuit for resonance maintaining is added. It is used to power the parallel resonance inductive furnace.

The practical use of the described solution has shown very good results. The system has been tested with different loads and permanent short-circuit. It is operating in the Mining and Metallurgy Institute in Bor.

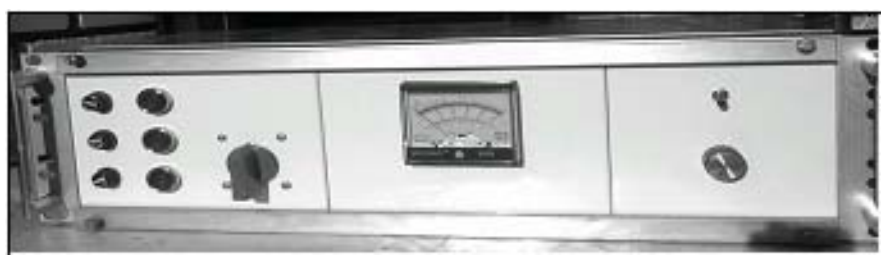

Fig.6 The picture of the created unit using a new control method

\section{ACKNOWLEDGMENT}

This paper is supported by the Grant of the Ministry of Science of Republic of Serbia, as a part of a project EE18016 within the framework of Technological development program.

\section{REFERENCES}

[1] Radetic, R. (2005). Prekidačka napajanja. Beograd: Nauka.

[2] Radetic, R. (2006). Tranzistorski pretvaraci. Bor: Radojle Radetic.

[3] Jang, Y., Jovanović, M. (2007). A new PWM ZVS fullbridge converter. IEEE Trans. Power Electron., 22 (3), 987-994.

[4] ISL6551 (2006). Intersil, Data Sheet. January 3, 2006, FN9066.5.

[5] Radetic, R.M., Milivojevic, D.R. (2009). RR complementary control method for transistor bridge converter. In International Symposium on Power Electronics - EE 2009, October 28-30, 2009. Novi Sad, Republic of Serbia. 